\title{
Sulfur mustard induces expression of metallothionein-IA in human airway epithelial cells
}

This article was published in the following Dove Press journal:

International Journal of General Medicine

25 May $201 \mathrm{I}$

Number of times this article has been viewed

\author{
Mohammad Reza Nourani' \\ Majid Ebrahimi' \\ Mehryar Habibi Roudkenar ${ }^{3}$ \\ Ensieh Vahedi' \\ Mostafa Ghanei' \\ Abbas Ali Imani Fooladi² \\ 'Chemical Injury Research Center; \\ ${ }^{2}$ Microbial Product Research Center, \\ Baqiyatallah University of Medical \\ Sciences; ${ }^{3}$ Research Center, Iranian \\ Blood Transfusion Organization, \\ Tehran, Iran
}

Background: Sulfur mustard can cause several long-term complications in the organs of individuals exposed to this toxic gas, and among these, pulmonary sequelae are the most important. More than 25 years after the Iran-Iraq war, thousands of Iranians are suffering from the chronic respiratory complications of sulfur mustard. Currently, based on several clinical findings, bronchiolitis obliterans is confirmed as the major diagnosis in these patients. Numerous studies have revealed that this disorder is strongly associated with oxidative stress due to excessive production of harmful reactive substances and decreased levels of endogenous antioxidants. Metallothioneins (MTs) are a group of low molecular weight sulfhydryl-rich intracellular proteins, and several isoforms have been identified in humans. MT-1A is an inducible and important MT isoform, which is transcriptionally activated by a variety of stress stimuli, such as free radicals

Methods: MT-1 mRNA expression and protein levels in endobronchial biopsy samples from 24 sulfur mustard-exposed patients and 15 unexposed control cases were evaluated by semiquantitative reverse transcriptase polymerase chain reaction, real-time reverse transcriptase polymerase chain reaction, and immunohistochemistry.

Results: mRNA- MT-1A expression levels in sulfur mustard-exposed patients were upregulated compared with normal samples. Protein expression was also markedly higher in controls than in sulfur mustard-exposed patients.

Conclusion: Upregulation of MT-1A mRNA in patients who have been exposed to sulfur mustard seems to be due to oxidative stress, which is induced in an attempt to ameliorate this harmful situation by reestablishment of homeostasis, but depletion of its protein might be due to secondary consequences of sulfur mustard toxicity, which are as yet not understood.

Keywords: sulfur mustard, metallothionein-1A, airway, epithelial cells

\section{Introduction}

Respiratory system sequelae are the most important long-term complications in patients exposed to sulfur mustard. Thousands of Iranian civilians and veterans are still suffering from delayed respiratory difficulties caused by inhalation of this toxic gas during the Iraq conflict with Iran more than 25 years ago.,

Cough, dyspnea, excessive sputum production, and hemoptysis are among the most prominent chronic pulmonary symptoms in sulfur mustard-injured patients. ${ }^{3,4}$ Nowadays, bronchiolitis obliterans is part of the diagnosis in these patients. ${ }^{5}$ However, a number of differences have been found between this kind of bronchiolitis obliterans and other kinds, such as that following lung transplantation, and there is no progression pattern, rare fibrosis, differing severity, and less obliteration. ${ }^{6}$ Investigations have
Correspondence: Abbas Ali Imani Fooladi Microbial Product Research Center Baqiyatallah University of Medical

Sciences, Tehran I4359-447II, Iran

$\mathrm{Tel} / \mathrm{fax}+982188211523$

Email imanifouladi.a@gmail.com 
shown that the pulmonary disorder in patients with chronic sulfur mustard exposure is strongly associated with oxidative stress, caused by an imbalance between excessive creation of reactive oxygen and/or nitrogen species and depletion of endogenous antioxidants, ${ }^{7}$ along with the release of several kinds of inflammatory mediators from a number of cell types. ${ }^{8,9}$ A recent study has shown that serum glutathione levels are significantly lower in patients exposed to sulfur mustard than in controls, but the level of malondialdehyde is significantly higher in sulfur mustard victims. ${ }^{10}$ More recently, in a study of airway biopsy samples, we demonstrated that expression of lipocalin-2 at the mRNA level was significantly higher in sulfur mustard-injured patients in comparison with controls, but there was no significant difference in the expression of lipocalin-2 protein between patients and controls. ${ }^{11}$ These data confirm that oxidative stress due to excessive production of harmful reactive substances and decreased levels of endogenous protective antioxidants is a phenomenon in these patients that plays a pivotal role in the pathology of late pulmonary complications of sulfur mustard exposure. However, the exact mechanisms still need to be elucidated.

Metallothioneins (MTs) are a group of low molecular weight $(6-7 \mathrm{kDa})$, sulfhydryl-rich intracellular proteins with 61 to 68 amino acid residues, initially recognized by Margoshes and Vallee in 1957 as cadmium-binding proteins in equine kidney tissue. In human beings, several MT isoforms, from MT-1 through MT-4, have been identified. ${ }^{12}$ MT-1 is an inducible MT isoform encoded by a group of more than 10 functional genes, amongst which MT-1A is a broadly disseminated MT isoform in the human body. Its transcription is activated by a variety of stress stimuli, including metals, glucocorticoids, a number of proinflammatory cytokines, and reactive oxygen species. ${ }^{13,14}$ Intracellular metal homeostasis, heavy metal detoxification, and scavenging of a wide variety of compounds, including hydroxyl radicals, superoxide, hydrogen peroxide, and nitric oxide, are among the accepted roles of MT-1, although the exact molecular mechanisms involved are not fully understood. ${ }^{15,16}$

A number of studies have demonstrated coexpression of MT-1 and lipocalin-2 in some illnesses, including oxidative stress-mediated lung injuries. ${ }^{17}$ The present study aimed to assess the expression of MT-1A at the mRNA and protein levels in bronchial biopsy samples of sulfur mustard-injured patients in comparison with controls. Our results may pave the way to new treatments for the chronic respiratory complications encountered in these patients.

\section{Material and methods Study design}

Twenty-four sufferers of chronic respiratory sequelae from exposure to sulfur mustard during the 1980-1988 Iran-Iraq war were enrolled as the sulfur mustard-exposed group and 15 unexposed individuals as the control group. All of the subjects were male. Contact with sulfur mustard was confirmed by documents from the Iranian military health services at the time of exposure. These victims all developed pulmonary symptoms immediately after contact with sulfur mustard, without any symptom-free periods. The study was approved by the ethics committee of the Baqiyatallah University of Medical Sciences. The procedures conformed to the guiding principles of the Declaration of Helsinki, and all subjects signed informed consent forms for participation in the study. Potential subjects having other interactive criteria, as well as those involving other chronic pulmonary diseases (eg, asthma), autoimmune disease (eg, rheumatoid arthritis), lung cancer, diabetes mellitus, acute infective bronchitis, or pneumonia, were excluded. Drug addicts, elderly people ( $>65$ years old), smokers, organ transplant recipients, and patients with a history of occupational pulmonary exposure to other toxic agents were also excluded. Age, gender, and pulmonary function test data for the two groups are shown in Table 1.

All subjects were anesthetized by inhalation of $2 \%$ aerosolized lidocaine and intravenous midazolam, and slept

Table I Characteristics and pulmonary function test results of SM-injured patient and control group

\begin{tabular}{|c|c|c|c|}
\hline & $\begin{array}{l}\text { Control group subjects } \\
n=15\end{array}$ & $\begin{array}{l}\text { SM-injured patients } \\
n=24\end{array}$ & $P$ value \\
\hline Age range & $22-57$ & $30-58$ & - \\
\hline Age (mean $\pm S D)$ & $43.6 \pm 10.92$ & $42.9 \pm 5.48$ & 0.83 \\
\hline $\mathrm{FVC}($ mean $\pm \mathrm{SD})$ & $3.34 \pm 0.79$ & $2.87 \pm 0.89$ & 0.11 \\
\hline $\mathrm{FEV}_{1}($ mean $\pm \mathrm{SD})$ & $2.7 \mathrm{I} \pm 0.8 \mathrm{I}$ & $1.92 \pm 0.87$ & $0.007^{*}$ \\
\hline $\mathrm{FEV}_{\mathrm{I}} / \mathrm{FVC}($ mean $\pm \mathrm{SD})$ & $79.85 \pm 6.15$ & $67.88 \pm 15.81$ & $0.00 I^{*}$ \\
\hline $\mathrm{RV}($ mean $\pm \mathrm{SD})$ & $2.38 \pm 0.97$ & $3.75 \pm 1.75$ & $0.04 *$ \\
\hline
\end{tabular}

Note: $* P<0.05$.

Abbreviations: FVC, forced vital capacity; $\mathrm{FEV}_{\text {, }}$, forced expiratory volume in I second; RV, residual volume; SD, standard deviation. 
lightly throughout the procedure. Bronchoscopy was carried out using a flexible fiberoptic bronchoscope (BF1T; Olympus, Tokyo, Japan) passed through the airway to reach the segmental and subsegmental carinae, and endobronchial biopsy specimens were taken from these regions using bronchoscopic forceps (Olympus). Supplemental oxygen was given throughout the procedure, and oxygen saturation was checked at regular intervals by a pulse oximeter until the subjects regained consciousness.

Two biopsy samples were taken from each patient, and were immediately and separately immersed in Tripure isolation reagent (Roche, Mannheim, Germany) and formalin (Merck, Darmstadt, Germany). The samples in Tripure were stored at $-80^{\circ} \mathrm{C}$ until RNA extraction, and the formalin samples were kept at $4^{\circ} \mathrm{C}$ for immunohistochemistry.

\section{Reverse transcriptase polymerase chain reaction analysis of MT-IA gene expression}

We have already described the reverse transcriptase polymerase chain reaction procedure used in this study. ${ }^{11}$ In brief, all the RNA contained in the airway biopsy specimens was harvested in Tripure isolation reagent in accordance with the manufacturer's protocol and kept at $-80^{\circ} \mathrm{C}$ during the procedure. The RNA extracted was evaluated by Nanodrop spectrophotometer (ND-1000; Wilmington, DE), and its quality was confirmed by electrophoresis in 1\% agarose gel (Cinnagen, Tehran, Iran). Aliquots of $500 \mathrm{ng}$ of isolated RNA were utilized as templates for cDNA synthesis by SuperScript III reverse transcriptase (Invitrogen, Carlsbad, CA) following the manufacturer's instructions.

Semiquantitative reverse transcriptase polymerase chain reaction for the MT-1A gene was carried out using equal amounts of synthesized cDNA, in a final reaction volume of $25 \mu \mathrm{L}$. All reagents and recombinant Taq DNA polymerase were obtained from Cinnagen, and the reactions were done in a master cycler thermal cycler. Specific primers for MT-1A and $\beta$-actin (as a housekeeping gene) were designed using primer3 software (http://frodo.wi.mit. $\mathrm{edu} /$ ) and ordered from Bioneer (Daejeon, South Korea, see Table 2). The polymerase chain reaction conditions comprised primary denaturation at $94^{\circ} \mathrm{C}$ for 5 minutes, followed by 30 polymerase chain reaction cycles comprising denaturation at $94^{\circ} \mathrm{C}$ for 30 seconds, annealing at $59^{\circ} \mathrm{C}$ (both genes at the same temperature) for 30 seconds, extension at $72^{\circ} \mathrm{C}$ for 60 seconds, followed by 5 minutes of terminal extension at $72^{\circ} \mathrm{C}$. Finally, the polymerase chain reaction products were electrophoretically separated in $2 \%$ agarose gel and dyed with ethidium bromide (Cinnagen). Bands were visualized under ultraviolet light in gel documentation (Bio-RadLaboratories, Hercules, CA).

Quantitative real-time reverse transcriptase polymerase chain reaction was then performed in a Rotor-Gene RG 3000 (Corbett Research, Mortlake, Australia). The amplification procedure, run in triplicate for each sample, used SYBR Green Premix (Takara Holdings, Inc, Shiga, Japan) according to the manufacturer's instructions. Quantitative polymerase chain reaction criteria comprised initial denaturation at $94^{\circ} \mathrm{C}$ for 1 minute, followed by 40 amplification cycles, including denaturation at $94^{\circ} \mathrm{C}$ for 20 seconds, annealing at $59^{\circ} \mathrm{C}$ for 30 seconds, and extension at $72^{\circ} \mathrm{C}$ for 30 seconds. $\beta$-actin gene expression was used to normalize threshold cycle values $\left(\mathrm{C}_{\mathrm{t}}\right)$ of the target gene, MT-1A, and provided us with a control for relative quantitative evaluation of the abundance of transcripts using the $2^{-\Delta \Delta \mathrm{CT}}$ method.

\section{Immunohistochemistry}

We have described the immunohistochemistry procedures used in this study in detail elsewhere. ${ }^{18}$ Briefly, 10 airway biopsy samples from sulfur mustard-injured patients and 10 specimens from unexposed controls were examined. All samples were fixed in 4\% formalin (Merck) and then immersed in phosphate-buffered saline (Takara Holdings, Inc) containing 30\% sucrose (Wako, Osaka, Japan). Water-embedded sections $15 \mu \mathrm{m}$ thick were prepared by cryostat (Histo-line, Milan, Italy), and incubated at $4^{\circ} \mathrm{C}$ with primary antibody at a dilution of 1:200 in phosphate-buffered saline for 12 hours. The primary antibody was a mouse monoclonal antibody raised against the human MT-1 isoform

Table 2 Sequence and features of PCR

\begin{tabular}{llll}
\hline Gene (gene bank ID) & Primer sequence $\left(5^{\prime}\right.$ to $\left.\mathbf{3}^{\prime}\right)$ & Annealing Tm ( $\left.{ }^{\circ} \mathbf{C}\right)$ & Product size (bp) \\
\hline $\begin{array}{l}\text { MT-IA (NM_005946) } \\
\quad \text { Forward: }\end{array}$ & ATGGACCCCAACTGCTCCTGC & 59 & 177 \\
$\quad$ Reverse: & CAGCTGCACTTCTCTGATGCCC & \\
$\begin{array}{l}\text { B-actin (NM_00II0I) } \\
\text { Forward: }\end{array}$ & TTCTACAATGAGCTGCGTGTGG & 59 & 119 \\
Reverse: & GTGTTGAAGGTCTCAAACATGAT & & \\
\hline
\end{tabular}


(Abcam, Cambridge, UK). Next, the sections were incubated with biotinylated antimouse secondary antibody (Santa Cruz Biotechnology, Santa Cruz, CA), and diluted to 1:200 for immunostaining. The sections were visualized using the mouse ABC staining system (Santa Cruz Biotechnology), and using 3,3'-diaminobenzidine as the substrate.

\section{Statistical analysis}

Data were calculated as mean \pm the standard deviation of fold-changes in MT-1A gene expression in three independent experiments. SPSS software (v 15.0; SPSS, Inc, Chicago, IL) was used for the statistical analyses. Student's $t$-test was used for the evaluation of differences in gene expression between the sulfur mustard-injured group and the unexposed group, and $P<0.05$ was considered to be statistically significant.

\section{Results}

In total, 39 subjects participated in this study, comprising 24 sulfur mustard-injured patients and 15 normal unexposed control individuals. The average age of the sulfur mustard-injured patients and the unexposed controls was not significantly different (42.9 versus 43.6 years, respectively, $P=0.83$, see Table 1$)$.

The results of pulmonary function testing are shown in Table 1. Although forced vital capacity in the control group was higher than in sulfur mustard-injured cases, the difference was not statistically significant $(P=0.11)$. On the other hand, forced expiratory volume in 1 second $\left(\mathrm{FEV}_{1}\right)$ in the sulfur mustard group was significantly lower than in the controls $(P=0.007)$. Moreover, $\mathrm{FEV}_{1} /$ forced vital capacity also differed between the two groups, being significantly higher in the controls $(P=0.001)$. Residual volume was significantly elevated in sulfur mustard-injured patients in comparison with controls $(P=0.43)$.
We initially used a semiquantitative reverse transcriptase polymerase chain reaction to elucidate whether there were any variations in MT-1A gene expression among the control samples, and our results revealed no significant differences (data not shown).

Next, we examined the expression of MT-1A in the sulfur mustard-injured patients. Because the controls had expressed identical levels of the gene, all of them were used for comparison with the results in the sulfur mustard-injured group. Our data revealed that MT-1A mRNA is upregulated in sulfur mustard-injured patients (Figure 1). The expression of MT-1A was also quantitatively evaluated by real-time reverse transcriptase polymerase chain reaction. The results showed that expression of this gene at the mRNA level is $4.0 \pm 2.60$ times higher in sulfur mustard-injured patients in comparison with control samples $(P=0.001$, see Table 3$)$.

Immunohistochemistry was employed for evaluation and localization of MT-1 protein expression in airway biopsy samples from the two groups. It was immediately observed that the thickness of the bronchial epithelium layer in sulfur mustard-exposed patients was about twice that in normal control biopsies. In the control group, strong MT-1 immunoreactivity was seen in airway epithelium cells, especially in the basal (germinal) layer (Figures 2A and 2C). In contrast, in sulfur mustard-injured specimens, very weak MT-1 protein expression was seen in the bronchial epithelial cells, especially at the luminal side of the brush border cells, indicating very low MT-1 protein expression (Figures 2B and 2D).

\section{Discussion}

Bronchiolitis obliterans is the major long-term pulmonary complication in Iranian victims of sulfur mustard exposure, ${ }^{5}$ but the exact molecular mechanisms involved in the symptoms observed need to be clarified. Air trapping in expiratory high-resolution computed tomography scans (data not shown)

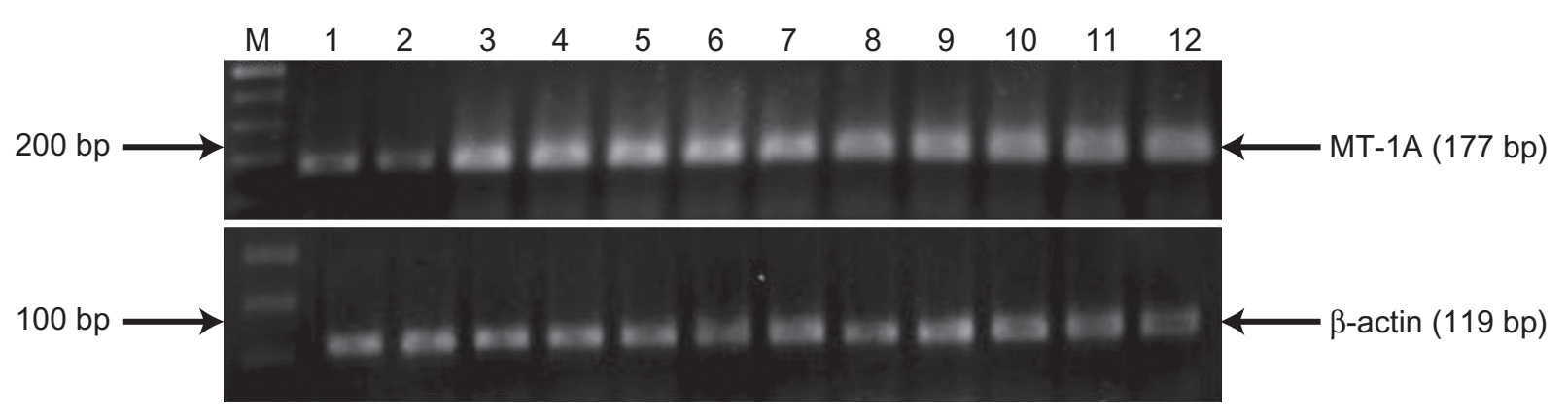

Figure I MT-IA gene expression in SM-injured patients and in unexposed control cases. Gene expressions were measured by semiquantitative reverse transcriptase polymerase chain reaction, and were upregulated in SM-injured patients (lanes 3-I2). Normal samples (lanes I and 2), SM-injured patients samples (lanes 3-I3). Abbreviations: SM, sulfur mustard; M, 100 bp marker; MT, metallothionein. 
Table 3 Increases in MTI A expression in sulfur mustard-exposed patients in comparison with control group

\begin{tabular}{lll}
\hline Case group/gene & $\begin{array}{l}\text { SM-exposed cases vs } \\
\text { unexposed cases }\end{array}$ & $\boldsymbol{P}$ (t-test) \\
\hline MT-IA & $4.0 \pm 2.60$ & $0.00 I^{*}$ \\
\hline
\end{tabular}

was the most common finding in our sulfur mustard-exposed patients. Pulmonary function test results showed an airflow obstruction pattern highly indicative of bronchiolitis obliterans as the major chronic pulmonary disorder in these patients.

Moreover, our semiquantitative reverse transcriptase polymerase chain reaction results, confirmed by quantitative realtime reverse transcriptase polymerase chain reaction, indicate that expression of MT-1A at the mRNA level was significantly elevated in bronchial biopsy samples from the sulfur mustardexposed group in comparison with the unexposed controls. As far as we are aware, very little is known about the precise molecular mechanisms involved in the structural modifications and pathophysiological symptoms of late complications of sulfur mustard exposure, and there are no published reports concerning MT-1A expression and its interaction with the delayed respiratory difficulties arising from sulfur mustard exposure.

A number of studies have shown that oxidative stress plays an important role in the pathogenesis of various lung

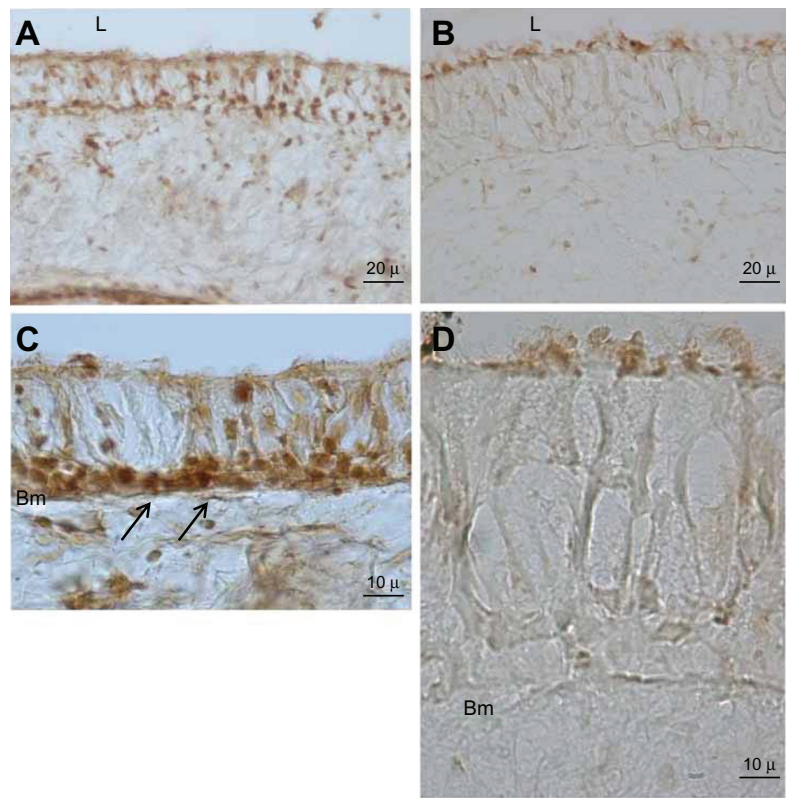

Figure 2 Immunohistochemical staining for MT-IA protein in bronchial epithelium. Left side micrographs reveal the expression of MT-IA in the basal cell layer of control group airway epithelium (arrow, $\mathbf{A}$ and $\mathbf{C}$ ). Right side micrographs show MT-IA expression in SM-injured airway epithelium (B and $\mathbf{D})$. Note the immunoreactivity of MT-AI in the control group is higher and wider than in the SM-injured samples. In the high and same magnification the epithelium layer thickness in SM-injured is much thicker than control group (C and $\mathbf{D})$.

Abbreviations: L, airway lumen; Bm, basement membrane; MT, metallothionein SM, sulfur mustard. diseases, including chronic obstructive pulmonary disease and asthma. ${ }^{20-21}$ Consistent with these findings, Ghanei et al ${ }^{22}$ have hypothesized that the long-term pulmonary complications of sulfur mustard exposure are neutrophil-dominant and/or lymphocyte-dominant disorders. It has also been shown that oxidative stress due to excessive production of reactive substances and depletion of endogenous antioxidants plays an important role in the pathogenesis of these delayed pulmonary sequelae. ${ }^{10,23}$ The recent finding that sulfur mustard increases endogenous production of reactive oxygen species suggests that reactive oxygen species are likely to be involved in the toxicity induced by this chemical warfare agent. ${ }^{7,8}$

The antioxidant activity of MT-1 was first demonstrated by Thornalley and Vasak, ${ }^{24}$ and it has since been proposed that MT-1 is involved in protection against damage caused by oxidative stress. Although the exact mechanism of the free radical scavenging action of MT-1 is not fully described, it has been postulated that MT- 1 cysteine residues can react directly as thiolate groups with a number of reactive substances, as well as with hydroxyl radicals, peroxyl radicals, superoxide hydrogen peroxide, and nitric oxide. ${ }^{25-27}$

Pitt et $\mathrm{al}^{28}$ demonstrated that overexpression of MT decreases the sensitivity of pulmonary endothelial cells to oxidative damage. They showed that pulmonary artery endothelial cells in sheep transfected by both human and mouse MT were more resistant to tert-butyl hydroperoxide, an oxidant-generating substance, and were also more resistant to hyperoxia. They suggested that MT can defend this cell type from attack by a variety of pro-oxidants. In another study, Wesselkamper et $\mathrm{al}^{29}$ showed that MT- $1 / 2$ transgenic mice had more resistance to nickel-induced acute lung injury than did MT-1/2 knockout mice, implying that MT can improve survival and inhibit progression of acute lung injury. More recently, an in vivo study by Helal and Helal ${ }^{30}$ demonstrated that intraperitoneal administration of MT can protect rats against carmustine-provoked lung injury because of its antioxidant attributes and its ability to suppress tumor necrosis factor alpha.

Upregulation of MT-1A mRNA in the airways of sulfur mustard-injured patients, in addition to oxidative stress, could be due to inflammation. Some in vivo experiments have demonstrated that a number of pro-inflammatory cytokines, as well as tumor necrosis factor alpha, interleukin (IL)-1, IL-6, and interferon gamma can induce MT gene expression. ${ }^{31-33}$ Depending on the site, stimulus direction, and type of pathophysiologic situation, MT can have diverse anti-inflammatory roles. ${ }^{34}$ Emad and Emad ${ }^{35}$ evaluated levels of several cytokines in bronchoalveolar lavage fluid from 
sulfur mustard-exposed patients and demonstrated that IL-8, IL-1 $\beta$, IL-6, IL-12, and tumor necrosis factor alpha levels in sulfur mustard-injured patients were all significantly higher than in controls, and suggested that neutrophilic pulmonary inflammation is possibly the mechanism underlying the long-term pulmonary complications of sulfur mustard exposure. Molecular level investigation of the existence of inflammation in the airways of sulfur mustard-injured patients is underway in our laboratory, and preliminary, as yet unpublished data show that tumor necrosis factor alpha expression at the mRNA level is significantly upregulated in sulfur mustard-exposed patients in comparison with controls, suggesting that inflammatory processes are involved in this chronic pulmonary complication. Consistent with these findings, Takano et $\mathrm{al}^{36}$ have shown that MT-1/2 knockout mice are more vulnerable to acute pulmonary inflammatory injury mediated by intratracheal instillation of lipopolysaccharide than wild-type mice. Additionally, in line with these findings, a complementary DNA microarray study based on acute inflammatory murine lung damage induced by lipopolysaccharide and diesel exhaust particles revealed that MT-1 gene expression at the mRNA level is significantly upregulated in mice exposed to these irritants. ${ }^{17}$ Similarly, a more recent study showed that MT plays an important role in ozone-induced pulmonary inflammation, and showed that lung inflammation is significantly greater in MT-1/2 knockout than wild-type mice. ${ }^{37}$ However, despite numerous investigations of the possible contribution of MT-1 to inflammatory lung injuries, its exact role still needs to be elucidated.

Paradoxically, our immunohistochemistry results revealed that, in contrast with the upregulation of MT-1A mRNA expression in airway biopsy samples from sulfur mustard-injured patients, its protein level was higher in control subjects. Several MT-1 immunoreactive cells were seen in the bronchial epithelia of our control cases, and immunoreactivity was more intense in cells adjacent to the basement membrane. This finding is in accordance with that of Courtade et al, ${ }^{38}$ who evaluated the expression of MT in the normal human lung, and observed positively stained pleural endothelial cells and basal cells from the bronchial epithelium. In contrast, in our chemically-injured cases, only a very weak expression of MT-1 was observed on the luminal side of the bronchial epithelium. We have also already shown an inconsistency between mRNA and protein expression of lipocalin-2 and heme oxygenase in the bronchial epithelium of sulfur mustard-exposed patients compared with unexposed cases. ${ }^{11,39,40}$ We hypothesize that this discrepancy between mRNA and protein expression of MT-1A may be caused by translational inefficiency and/or posttranslational regulation. Interestingly, it has been revealed that, despite a huge amount of evidence strongly suggesting that the expression of MT genes is transcriptionally regulated, new data have also indicated that posttranslational modulations also have effects on MT gene expression. ${ }^{14}$ This discrepancy may be due to a change in the expression of microRNAs, which are posttranscriptional regulators that bind to complementary sequences on target messenger RNA transcripts, usually resulting in translational repression and gene silencing. ${ }^{41}$

Intriguingly, it has been shown that administration of $\mathrm{N}$-acetylcysteine can significantly improve clinical symptoms in sulfur mustard-exposed patients, ${ }^{19}$ and it has also been demonstrated that N-acetylcysteine, as an antioxidant, provides thiol groups which are essential for serum glutathione production. ${ }^{39}$ In this way, $\mathrm{N}$-acetylcysteine can compensate for decreased expression of MT-1 protein, but, in spite of this improvement, no cure has as yet been achieved in sulfur mustard-injured patients.

\section{Conclusion}

This study shows that although the expression of MT-1 at the mRNA level was significantly increased in the bronchial epithelium of sulfur mustard-exposed patients, expression of MT-1 protein was significantly higher in the airways of our controls. This upregulation of MT-1 mRNA seems to be due to oxidative stress and depletion of the endogenous antioxidants which exist in the lungs of patients who have inhaled sulfur mustard, and may reflect an attempt to ameliorate this harmful situation by reestablishment of homeostasis. The contrasting depletion of its protein may be a result of secondary effects of sulfur mustard toxicity. Therefore, further studies clarifying the mechanisms involved in the long-term outcome of sulfur mustard exposure are warranted.

\section{Acknowledgment}

We thank the members of our laboratory at the Chemical Injury Research Center, Baqiyatallah University of Medical Sciences for their contributions to this research.

\section{Disclosure}

The authors report no conflicts of interest in this work.

\section{References}

1. Ghanei M, Aslani J, Khateri S, Hamadanizadeh K. Public health status of the civil population of Sardasht 15 years following large-scale war time exposure to sulfur mustard, 2003. J Burns Surg Wound Care. 2003;2:7-18.

2. Khateri S, Ghanei M, Soroush M, Haines D. Incidence of lung, eye and skin lesions as late complications in 34,000 Iranians with wartime exposure to mustard agent. J Occup Environ Med. 2003;452:1136-1143. 
3. Emad A, Rezaian GR. The diversity of the effects of sulfur mustard gas inhalation on respiratory system 10 years after a single, heavy exposure. Chest. 1997;112:734-730.

4. Balali-Mood M, Hefazi M. Comparison of early and late toxic effects of sulfur mustard in Iranian veterans. Basic Clin Pharmacol Toxicol. 2006;99:273-282.

5. Ghanei M, Mokhtari M, Mohammad MM, Aslani J. Bronchiolitis obliterans following exposure to sulfur mustard: chest high resolution computed tomography. Eur J Radiol. 2004;52:164-169.

6. Ghanei M, Tazelaar HD, Chilosi M, et al. An international collaborative pathologic study of surgical lung biopsies from mustard gas exposed patients. Respir Med. 2008;102:825-830.

7. Han S, Espinoza LA, Liao H, Boulares AH, Smulson ME. Protection by antioxidants against toxicity and apoptosis induced by the sulphur mustard analog 2-chloroethylethyl sulphide (CEES) in Jurkat T cells and normal human lymphocytes. Br J Pharmacol. 2004;141:795-802.

8. Korkmaz A, Yaren Y, Topal T, Oter S. Molecular targets against mustard toxicity: Implication of cell surface receptors, peroxynitrite production, and PARP activation. Arch Toxicol. 2006;80:662-670.

9. Toews GB. Impact of bacterial infections on airway diseases. Eur Respir Rev. 2005;14:62-68.

10. Shohrati M, Ghanei M, Shamspour N, et al. Glutathione and malondialdehyde levels in late pulmonary complications of sulfur mustard intoxication. Lung. 2010;188:77-83.

11. Ebrahimi M, Roudkenar MH, Imani Fooladi AA, et al. Discrepancy between mRNA and protein expression of neutrophil gelatinaseassociated lipocalin in bronchial epithelium induced by sulfur mustard J Biomed Biotechnol. 2010;2010:823131.

12. Thirumoorthy N, Manisenthil Kumar KT, Sundar SA, et al. Metallothionein An overview. World J Gastroenterol. 2007;13:993-996.

13. Lu H, Hunt DM, Ganti R, et al. Metallothionein protects human retinal pigment epithelial cells aganist apoptosis and oxidative stress. Exp Eye Res. 2002;74:83-92.

14. Haq F, Mahoney M, Koropatnick J. Signaling events for metallothionein induction. Mutat Res. 2003;533:211-226.

15. Davis SR, Cousins RJ. Metallothionein expression in animals: A physiological perspective on function. J Nutr. 2000;130:1085-1088.

16. Sato M, Kondoh M. Recent studies on metallothionein: Protection against toxicity of heavy metals and oxygen free radicals. Tohoku $J$ Exp Med. 2002;196:9-22.

17. Yanagisawa R, Takano H, Inoue K, et al. Complementary DNA microarray analysis in acute lung injury induced by lipopolysaccharide and diesel exhaust particles. Exp Biol Med (Maywood). 2004;229:1081-1087.

18. Nourani MR, Owada Y, Kitanaka N, et al. Localization of epidermaltype fatty acid binding protein in macrophages in advanced atretic follicles of adult mice. J Mol Histol. 2005;36:391-400.

19. Ghanei M, Aslani J, Khateri S, Hamadanizadeh K. Public health status of the civil population of Sardasht 15 years following large-scale war time exposure to sulfur mustard. J Burns Wounds.2003;2:7.

20. Greene L. Asthma, oxidant stress and diet. Nutrition. 1999;15:899-907.

21. Rahman I, Adcock IM. Oxidative stress and redox regulation of lung inflammation in COPD. Eur Respir J. 2006;28:219-242.

22. Ghanei M, Shohrati M, Jafari M, Ghaderi S, Alaeddini F, Aslani J. $\mathrm{N}$-acetylcysteine improves the clinical conditions of mustard gas-exposed patients with normal pulmonary function tests. Basic Clin Pharmacol Toxicol. 2008; 10:428-432.
23. Naghii MR. Sulfur mustard intoxication, oxidative stress, and antioxidants. Mil Med. 2002;167:573-575.

24. Thornalley PJ, Vasak M. Possible role for metallothionein in protection against radiation-induced oxidative stress. Kinetics and mechanism of its reaction with superoxide and hydroxyl radicals. Biochim Biophys Acta. 1985;827:36-44.

25. Kumari MV, Hiramatsu M, Ebadi M. Free radical scavenging actions of metallothionein isoforms I and II. Free Radic Res. 1998;29:93-101.

26. Min KS, Nishida K, Onosaka S. Protective effect of metallothionein to ras DNA damage induced by hydrogen peroxide and ferric ionnitrilotriacetic acid. Chem Biol Interact. 1999;122:137-152.

27. Zangger K, Shen G, Oz G, Otvos JD, Armitage IM. Oxidative dimerization in metallothionein is a result of intermolecular disulphide bonds between cysteines in the alpha-domain. Biochem J. 2001;359: 353-360.

28. Pitt BR, Schwarz M, Woo ES, et al. Overexpression of metallothionein decreases sensitivity of pulmonary endothelial cells to oxidant injury. Am J Physiol. 1997;273:L856-L865.

29. Wesselkamper SC, McDowell SA, Medvedovic M, et al. The role of metallothionein in the pathogenesis of acute lung injury. Am J Respir Cell Mol Biol. 2006;34:73-82.

30. Helal GK, Helal OK. Metallothionein attenuates carmustine-induced oxidative stress and protects against pulmonary fibrosis in rats. Arch Toxicol. 2009;83:87-94.

31. De SK, McMaster MT, Andrews GK. Endotoxin induction of murine metallothionein gene expression. J Biol Chem. 1990;265: $15267-15274$

32. Sato M, Sasaki M, Hojo H. Tissue specific induction of metallothionein synthesis by tumor necrosis factor-alpha. Res Commun Chem Pathol Pharmacol. 1992;75:159-172.

33. Waelput W, Broekaert D, Vandekerckhove J, Brouckaert P, Tavernier J, Libert C. A mediator role for metallothionein in tumor necrosis factorinduced lethal shock. J Exp Med. 2001;194:1617-1624.

34. Inoue K, Takano H, Shimada A, Satoh M. Role of metallothionein in inflammatory lung diseases. Curr Respir Med Rev. 2009;5:6-11.

35. Emad A, Emad Y. CD4/CD8 ratio and cytokine levels of the BAL fluid in patients with bronchiectasis caused by sulfur mustard gas inhalation. J Inflamm (Lond). 2007;4:2.

36. Takano H, Inoue $\mathrm{K}$, Yanagisawa $\mathrm{R}$, et al. Protective role of metallothionein in acute lung injury induced by bacterial endotoxin. Thorax. 2004;59:1057-1062.

37. Inoue $\mathrm{K}$, Takano H, Kaewamatawong $\mathrm{T}$, et al. Role of metallothionein in lung inflammation induced by ozone exposure in mice. Free Radic Biol Med. 2008;45:1714-1722.

38. Courtade M, Carrera G, Paternain JL, et al. Metallothionein expression in human lung and its varying levels after lung transplantation. Chest. 1998;113:371-378.

39. Dekhuijzen PNR. Antioxidant properties of N-acetylcysteine: Their relevance in relation to chronic obstructive pulmonary disease. Eur Respir J. 2004;23:629-636.

40. Nourani MR, Yazdani S, Roudkenar MH, et al. HO1 mRNA and protein do not change in parallel in bronchial biopsies of patients after long term exposure to sulfur mustard, gene regulation and systems biology. Gene Regul Syst Bio. 2009;4:83-90.

41. Bartel DP. MicroRNAs: Target recognition and regulatory functions Cell. 2009;136:215-233.
International Journal of General Medicine

\section{Publish your work in this journal}

The International Journal of General Medicine is an international, peer-reviewed open-access journal that focuses on general and internal medicine, pathogenesis, epidemiology, diagnosis, monitoring and treatment protocols. The journal is characterized by the rapid reporting of reviews, original research and clinical studies across all disease areas.

\section{Dovepress}

A key focus is the elucidation of disease processes and management protocols resulting in improved outcomes for the patient.The manuscript management system is completely online and includes a very quick and fair peer-review system. Visit http://www.dovepress.com/ testimonials.php to read real quotes from published authors. 